\title{
Resonant Behavior in Soft X-Ray Fluorescence Excited by Monochromatized Synchrotron Radiation
}

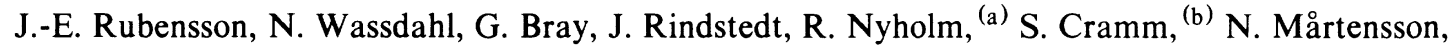 \\ and J. Nordgren \\ Department of Physics, Uppsala University, S-75121 Uppsala, Sweden
}

(Received 10 December 1987)

\begin{abstract}
Soft x-ray emission spectra of TiN, excited by monochromatized synchrotron radiation, have been recorded in a grazing-incidence spectrometer. The tunability of the excitation energy has allowed the pure $\mathrm{N} K$ and Ti $L_{\mathrm{III}}$ emission spectra to be isolated for the first time. A new type of resonance is observed in the Ti $L$ emission spectrum, and interpreted as due to the decay of a quasiatomic intermediate state. The results call for a reinterpretation of Ti $L$ spectra and a revision of the interpretation of $3 d$ metal $L$-emission spectra in general.
\end{abstract}

PACS numbers: 78.70. En

Up to the present date, soft $x$-ray emission spectroscopy has been pursued with less selective means of excitation. For intensity reasons and simplicity, most experiments have been performed with electron excitation, ${ }^{1-3}$ sometimes allowing selectivity in terms of an upper energy limit. ${ }^{4-6}$ For photon excitation, either broad-band synchrotron radiation, ${ }^{7,8}$ bremsstrahlung, ${ }^{9}$ or discrete $\mathrm{x}$ ray sources ${ }^{10,11}$ have been used. However, nonselectivity in the excitation often leads to problems in the interpretation of $x$-ray spectra, due to satellites and overlapping transition involving irrelevant core holes.

In the $\mathrm{x}$-ray region, one has already demonstrated the usefulness of monochromatized synchrotron radiation for excitation of fluorescence in gas-phase samples. ${ }^{12} \mathrm{As}$ is shown in the present work, recent progress in grazingincidence instrumentation ${ }^{13}$ and synchrotron radiation technique $^{14}$ has allowed narrow-band-pass photonexcited fluorescence spectroscopy also in the subkiloelectronvolt region. In the first series of experiments this achievement has been used to investigate the electronic structure of TiN. ${ }^{15}$ The tunability of the excitation energy has been used to isolate the $\mathrm{N} K$ emission spectrum from overlapping $\mathrm{Ti}$ intensity, as well as to separate the Ti $L_{\text {II }}$ and $L_{\text {III }}$ emission spectra. The results clearly show that the former interpretation, based on nonselectively excited spectra, must be revised. A spectral feature which has previously been assigned to $L_{\text {II }}$ emission is shown to be an $L_{\text {III }}$ satellite. The satellite intensity shows a resonant excitation-energy dependence, which is discussed in terms of quasiatomic excitations.

The emission was excited by photons from the Flipper I monochromator (band pass: $3 \mathrm{eV}$ ) at the multipole wiggler beam line at HASYLAB in Hamburg, ${ }^{14}$ and analyzed with a recently constructed portable grazingincidence spectrometer. ${ }^{13}$ In the present experiment the instrument uses a spherical grating with 5-m radius and 1200 grooves $/ \mathrm{mm}$, mounted at $88.1^{\circ}$ angle of incidence. The entrance-slit width was chosen to yield $2-\mathrm{eV}$ resolution for the $\mathrm{Ti} L$ recordings and $0.4-\mathrm{eV}$ resolution for the N $K$ recordings. The detector is based on CsI-coated multichannel plates and resistive anode readout technique. The self-absorption losses in the $\mathrm{Ti}$ spectra are estimated to be less than $20 \% .^{16}$

In Fig. 1(a) we show the 410-eV-excited $\mathrm{N} K$ emission spectrum, ${ }^{19}$ aligned to the Fermi level by means of the $\mathrm{x}$-ray photoemission spectroscopy (XPS) binding energy, 397.2 eV. ${ }^{20}$ No spectral changes are discernible as the excitation energy is lowered to $400 \mathrm{eV}$, indicating that the spectrum is free from satellites originating from multiple excitations. The spectrum consists of a main

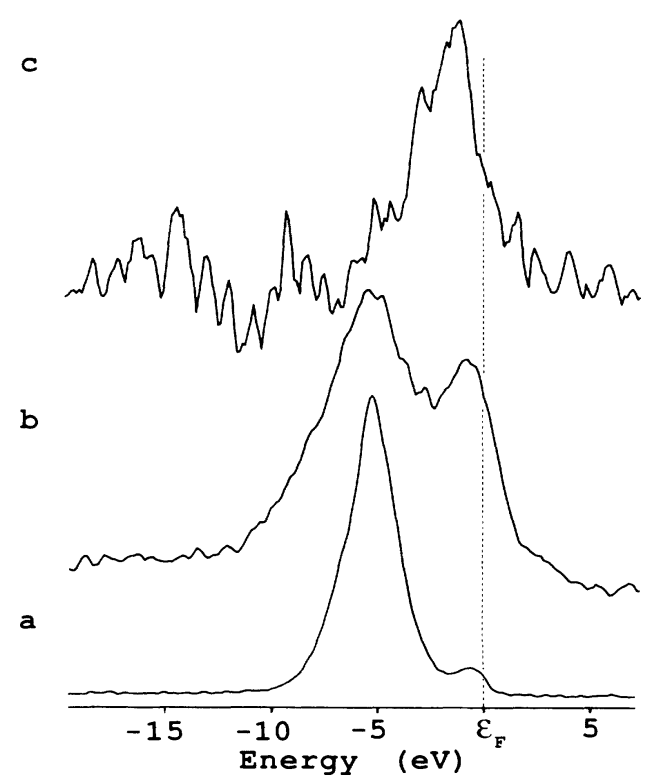

FIG. 1. The (a) $\mathrm{N} K$ and (b) $\mathrm{Ti} L$ emission spectra of $\mathrm{TiN}$, excited with $410-$ and $458-\mathrm{eV}$ photons, respectively. They are aligned to the Fermi energy by means of XPS core-electron binding energies. (c) The difference between the 480- and 458-eV-excited $\mathrm{Ti} L$ emission spectra normalized with respect to the low-energy region. The spectrum is aligned with the $2 p_{1 / 2}$ binding energy, determined from the $2 p_{3 / 2}$ XPS value and 6.1-eV spin-orbit splitting (Refs. 17 and 18) and it clearly deviates from the expected $L_{\text {II }}$ emission profile. 
band with a FWHM of $2.8 \mathrm{eV}$ situated $5.1 \mathrm{eV}$ below $\epsilon_{\mathrm{F}}$. There is a low-energy shoulder, and a high-energy band close to the Fermi level.

The Ti $L$ emission spectrum, excited with photons of energy below the $L_{\text {II }}$ threshold, is shown in Fig. 1 (b). It is free from $L_{\text {II }}$ emission and aligned to the Fermi level by employment of the $2 p_{3 / 2}$ ionization potential, 455.2 eV. ${ }^{17,20}$ The same two features are seen in the Ti $L$ spectrum as in the $\mathrm{N} K$ spectrum. ${ }^{21}$ However, in this case the peak at the Fermi level is much more pronounced.

The Ti $L$ and $\mathrm{N} K$ emission spectra mainly extract the Ti $3 d$ and $\mathrm{N} 2 p$ valence electron states, respectively. It is seen that the feature at $5 \mathrm{eV}$ is largely of $\mathrm{N} 2 p$ character, although it has also a considerable $\mathrm{Ti} 3 d$ contribution. The structure close to the Fermi level, however, is almost entirely of $\mathrm{Ti} 3 d$ character. This view is well supported by band-structure calculations, ${ }^{22}$ which give a set of three totally filled strongly $p$ - $d$ hybridized bands below a narrow Ti $3 d$ band populated by one electron.

In the $\mathrm{Ti} L$ emission spectra, excited well above the $L_{\text {II }}$ threshold, a third peak is discerned at higher $\mathrm{x}$-ray energies (see Fig. 2). The spectra excited at 473 and $480 \mathrm{eV}$ are similar and do not differ significantly from highenergy-excited spectra which have been interpreted as due to overlapping intensity from the $2 p_{3 / 2}$ and $2 p_{1 / 2}$ initial states. ${ }^{23,24}$ Our results are not compatible with this interpretation. Subtraction of the pure $L_{\text {III }}$ emission from spectra excited well above threshold does not yield the expected $L_{\text {II }}$ emission profile [Fig. 1(c)], which should be similar to the pure $L_{\text {III }}$ spectrum. ${ }^{25}$ The absence of the $L_{\text {II }}$ spectrum can be explained by a fast Coster-Kronig decay channel depopulating the $L_{\text {II }}$ state. From a comparison with $\mathrm{Ti}$ metal it is seen that this mechanism indeed should be important. ${ }^{26}$ To facilitate an alternative interpretation of the observed structure we

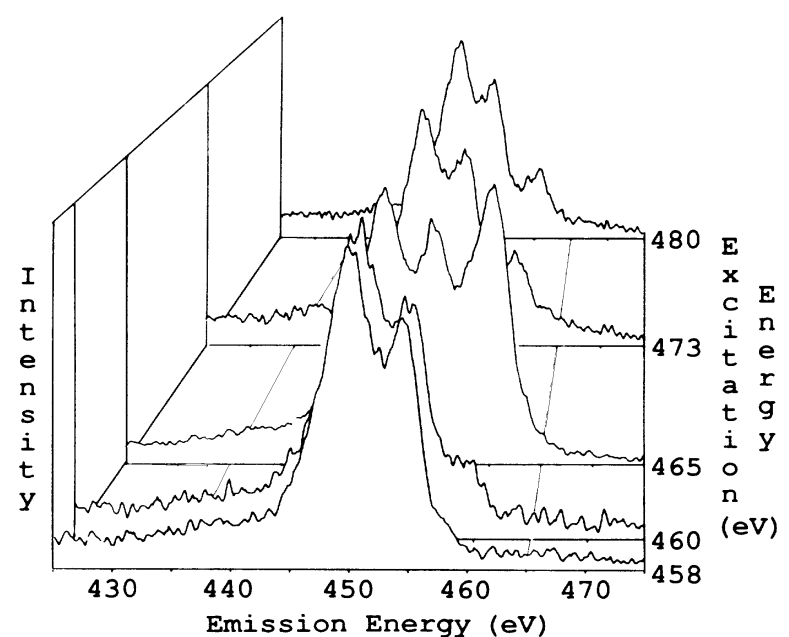

FIG. 2. Ti $L$ emission spectra of $\mathrm{TiN}$, excited at various photon energies, showing the resonant behavior of the $460.5-\mathrm{eV}$ feature. No corrections for self-absorption have been made. first turn our attention to the resonant behavior of the $\mathrm{Ti}$ $L$ emission.

Ti $L$ emission spectra excited with photons in the 458-480-eV energy range are shown in Fig. 2. The two main bands are situated at 449.9 and $454.4 \mathrm{eV}$, and as the energy is raised above $458 \mathrm{eV}$ a third peak appears at $460.5 \mathrm{eV}$ and assumes maximum intensity near $465-\mathrm{eV}$ excitation energy. As the energy is further increased the intensity of this peak decreases, but some intensity remains and the spectra excited at 473 and $480 \mathrm{eV}$ do not differ significantly from each other.

It has previously been shown that one-particle theories fail to describe the $L$ absorption spectra of the light $3 d$ metals. $^{27-30}$ The $L_{\text {II }} / L_{\text {III }}$ ratio is much larger than statistically expected, the absorption profile does not map the density of states above the Fermi level but is enhanced away from threshold, and the apparent spinorbit splitting does not agree with the XPS value. These effects have been accounted for by the inclusion also of atomic effects, the $2 p-3 d$ exchange interaction being the most important. ${ }^{29}$ Similar observations have been made in the electron energy-loss spectrum of TiN. ${ }^{31}$

This leads us to a tentative interpretation of the emission resonance in terms of quasiatomic excitations. The resonant feature reaches maximum intensity when the excitation energy is tuned to the correlation-enhanced absorption maximum associated with the $L_{\text {II }}$ level. The resonant emission energy is, however, about $5 \mathrm{eV}$ less than the excitation energy. To account for these observations we propose that a $2 p_{1 / 2}^{-1} 3 d$-like intermediate state (the notation indicates an atomiclike coupling between the $2 p$ hole and the $3 d$ electron) is excited and quickly depopulated via Coster-Kronig processes. The resulting quasiatomic $2 p_{3 / 2}^{-1} 3 d$ state subsequently decays, giving rise to the resonant $x$-ray emission satellite. The appearance of this satellite on the high-energy side is related to the localization of the $3 d$ electron (energy-gain satellite).

With above-resonance excitation energies, the $2 p^{-1} 3 d$ states can be populated by shakeup processes which cause the observed retention of the atomiclike characteristics of the spectrum.

The suggested qualitative interpretation reproduces the experimental results. Identification of the precise nature of the quasiatomic levels involved, regarding multiplets, transition probabilities, and energies, is beyond the scope of this investigation. Our results call for the reinterpretation of $\mathrm{Ti} L$ spectra of titanium compounds in general, where similar structures are observed in nonselectively excited spectra. ${ }^{23}$ Very likely these structures originate from similar physical processes, which we expect to be of importance also for other $3 d$ transition metals.

In the present context it is also interesting to note that anomalous $L V V$ Auger energies have been reported for the light $3 d$ transition metals. ${ }^{32,33}$ The Auger spectra have been found to yield two-hole spectra which are 
shifted to higher kinetic energies compared to the selfconvolution of the valence electron density of states. This has been interpreted as due to an attractive interaction between the two final $d$ holes (so-called negative $U),{ }^{32}$ or by a core-hole enhancement of the emission spectrum near the Fermi leve. ${ }^{33}$ In the light of the observed x-ray satellites, indicating core holes with additional excitations, we propose as an alternative explanation for the anomalous Auger spectra that the shifts to higher energies are due to the overlapping decay of more atomiclike excited core-hole states.

In conclusion, we have presented the soft $x$-ray emission spectra of TiN excited with monochromatized synchrotron radiation, and demonstrated some of the advantages of this technique. The Ti $L$ emission spectra show resonant behavior as the excitation energy is varied above the $L_{\mathrm{II}}$ threshold. We explain this behavior in terms of atomiclike excitations, though a detailed description calls for a more thorough theoretical investigation. The $\mathrm{N} K$ and Ti $L_{\text {III }}$ emission spectra, free from satellites and overlapping intensity, agree well with calculated partial density-of-states predictions.

We are indebted to HASYLAB for putting excellent research facilities at our disposal, in particular the Flipper I experimental station which was built with support from Bundesministerium für Forschung und Technologie under Grant No. 05-305-AX-B/3-KU. Professor C. Kunz is thanked for support and valuable discussions. We thank the solid state optics group of Uppsala University for providing the samples. This work was supported by the Swedish Natural Science Research Council.

(a) Permanent address: MAX Laboratory, Lund University, P. O. Box 118, S-22100 Lund, Sweden.

(b) Permanent address: Institut für Experimentalphysik, Universität Hamburg, 2000 Hamburg 50, Federal Republic of Germany.

${ }^{1}$ Soft X-ray Band Spectra, edited by D. J. Fabian (Academic, New York, 1968).

${ }^{2}$ Advances in X-ray Band Spectroscopy, edited by C. Bonnelle and C. Mande (Pergamon, New York, 1982).

${ }^{3}$ J. Nordgren and C. Nordling, Comments At. Mol. Phys. 13, 229 (1983).

${ }^{4}$ R. J. Liefeld, in Ref. 1, p. 133.

${ }^{5}$ C. Bonnelle, J. Phys. (Paris) 28, C3-65 (1967).

${ }^{6} \mathrm{~J}$.-J. Bonnet, D. Hubert, F. Bonnet, M. Bonnefoy, A. Fleury, and L. Awan, J. Phys. B 13, L187-191 (1980).

${ }^{7}$ E. Tegeler, E. Wiech, and A. Faessler, J. Phys. B 13, 4771 (1980).

${ }^{8}$ T. A. Callcott, K. L. Tsang, C. H. Zhang, D. L. Ederer, and E. T. Arakawa, Rev. Sci. Instrum. 57, 2680 (1986).

${ }^{9}$ E. Gilberg, M. J. Hanus, and B. Foltz, Jpn. J. Appl. Phys. 17, Suppl. 17-2, 101 (1978).

${ }^{10}$ B. L. Henke, R. C. C. Perera, E. M. Gullikson, and M. L. Schattenburg, J. Appl. Phys. 49, 480 (1978).

${ }^{11}$ G. Andermann, B. L. Henke, D. S. Urch, and G. Wiech, Jpn. J. Appl. Phys. 17, Suppl. 17-2, 428 (1978).
${ }^{12}$ R. C. C. Perera, R. E. LaVilla, P. L. Cowan, T. Jach, and B. Karlin, Phys. Scr. 36, 132 (1987).

${ }^{13} \mathrm{~J}$. Nordgren and R. Nyholm, Nucl. Instrum. Methods A 246, 242 (1986).

${ }^{14}$ F. Senf, K. Berens v. Rautenfeldt, S. Cramm, C. Kunz, J. Lamp, V. Saile, J. Schmidt-May, and J. Voss, Nucl. Instrum. Methods A 246, 314 (1986).

${ }^{15}$ The electronic structure of the transition-metal compounds has long been the subject of extensive investigations [J.-L. Calais, Adv. Phys. 26, 847 (1977); A. Neckel, Int. J. Quantum Chem. 23, 1317 (1983)]. Titanium nitrides and carbides are of considerable technical and theoretical interest since they possess an unusual combination of properties. While the melting point, hardness, and brittleness are representative of covalent crystals, the magnetic and electrical properties are typically metallic. The electronic structure of $\mathrm{TiN}$ has been explored by numerous experimental techniques, including $\mathrm{x}$-ray and $\mathrm{uv}$ photoelectron spectroscopy [L. I. Johansson, P. M. Stefan, M. L. Schek, and A. N. Christensen, Phys. Rev. B 22, 1032 (1980); L. Ramqvist, K. Hamrin, G. Johansson, A. Fahlman, and C. Nordling, J. Phys. Chem. Solids 30, 1835 (1969); L. Ramqvist, B. Ekstig, E. Källne, E. Noreland, and R. Manne, J. Phys. Chem. Solids 30, 1849 (1969); L. I. Johansson, A. L. Hagström, B. E. Jacobsson, and S. B. M. Hagström, J. Electron Spectrosc. Relat. Phenom. 10, 259 (1977); D. Denly, R. S. Williams, P. Perfetti, D. A. Shirley, and J. Stöhr, Phys. Rev. B 19, 1762 (1979)] and soft x-ray emission spectroscopy [D. W. Fischer and W. Baun, J. Appl. Phys. 39, 4757 (1968); V. A. Gubanov and E. Z. Kurmaev, Int. J. Quantum Chem. Symp. 9, 297 (1975); A. Z. Menshikov, I. A. Brytov, and E. Z. Kurmaev, Phys. Status Solidi 35, 89 (1969)] as well as by theoretical band-structure methods [A. Neckel, K. Schwartz, R. Eibler, P. Rastl, and P. Weinberger, Microchim. Acta, Suppl. 6, 257 (1975)].

${ }^{16}$ The exciting beam hit the sample at $10^{\circ}$ grazing angle, and the emission take-off angle was $80^{\circ}$. Geometrical considerations give the self-absorbed intensity

$$
A=A_{0}\left[1+\left(\mu_{0} / \mu_{i}\right) \tan 10^{\circ}\right]^{-1},
$$

where $A_{0}$ is the unperturbed intensity, and $\mu_{1}$ and $\mu_{0}$ are the absorption coefficients for the incident and outgoing radiation, respectively. We employ values derived from absorption measurements on Ti metal (Denly, Williams, Perfetti, Shirley, and Stöhr, Ref. 15).

${ }^{17}$ J. Fuggle and N. Mårtensson, J. Electron Spectrosc. Relat. Phenom. 21, 275 (1980).

${ }^{18}$ A. Lebugle, U. Axelsson, R. Nyholm, and N. Mårtensson, Phys. Scr. 23, 825 (1981).

${ }^{19}$ If the energy of the exciting radiation exceeds the Ti $2 p$ binding energy $(455 \mathrm{eV})$, the $\mathrm{N} K$ emission is distorted by the partly overlapping $\mathrm{Ti} 3 s-2 p$ emission. Attempts to overcome this difficulty have been made by Menshikov, Brytov, and Kurmaev (Ref. 15) and by I. A. Brytor, M. A. Rumsh, and A. S. Parobets, Fiz. Tverd. Tela (Leningrad) 10, 794 (1968) [Sov. Phys. Solid State 10, 621 (1968)], who constructed the N $K$ emission spectrum by subtracting the known line shape of the $3 s-2 p$ transition in titanium oxide. Also, the $\mathrm{N} K$ emission of $\mathrm{ZrN}$ has been taken to approximate that of TiN \{I. N. Frantsevich, E. A. Zhurakovskii, and N. N. Vasilenko, Dokl. Akad. Nauk. SSSR 198, 1066 (1971) [Sov. Phys. Dokl. 16, $481(1971)]\}$. 
${ }^{20}$ A. Lebugle, R. Nyholm, and N. Mårtensson, J. LessCommon Met. 82, 269 (1981).

${ }^{21}$ In the Ti $L$ emission spectrum there is a small bump on the high-energy slope, probably related to the peak appearing at higher excitation energies. The pertinent levels are reached because of the bandwidth of the exciting radiation. Also, a faintly increased background is discerned at lower energies, possibly consisting of satellites associated with excited final states.

${ }^{22}$ Neckel, Schwartz, Eibler, Rastl, and Weinberger, Ref. 15.

${ }^{23}$ Fischer and Baun, Ref. 15.

${ }^{24}$ Gubanov and Kurmaev, Ref. 15.

${ }^{25}$ D. A. Goodings and R. Harris, J. Phys. C 2, 1808 (1969).

${ }^{26}$ The $\mathrm{x}$-ray emission ratio is closely connected to the Auger and Coster-Kronig decay rates analogously to the Auger intensity ratio [E. Antonides, E. C. Janse, and G. A. Sawatzky, Phys. Rev. B 15, 4596 (1977); R. Nyholm, N. Mårtensson, A. Lebugle, and U. Axelsson, J. Phys. F 11, 1727 (1981)]. Assuming statistical population and equal Auger decay rates for the two holes, and that the emission from the directly excited and the Coster-Kronig populated $L_{\text {III }}$ holes fully overlap, we get the $L_{\mathrm{II}} / L_{\mathrm{III}}$ intensity ratio

$$
I=\frac{\Gamma_{2}}{\Gamma_{3}} \frac{\Gamma_{\mathrm{A}}}{3 \Gamma_{\mathrm{CK}}+2 \Gamma_{\mathrm{A}}},
$$

where $\Gamma_{\mathrm{CK}}, \Gamma_{\mathrm{A}}$, and $\Gamma_{i}$ are the Coster-Kronig, Auger, and $L_{i}$ emission lifetime widths, respectively. For $\mathrm{Ti}$ metal the broadenings are $\Gamma_{\mathrm{A}}=0.1 \mathrm{eV}$ and $\Gamma_{\mathrm{CK}}=0.4 \mathrm{eV}$ (Nyholm et al.). Using these values and assuming $\Gamma_{2}=\Gamma_{3}$ yields an intensity ratio $I=0.07$. Since $\mathrm{TiN}$ is metallic and has a large fraction of its $d$ density of states rather close to the Fermi level it is reasonable to assume similar rates in the compound.

${ }^{27}$ R. D. Leapman, L. A. Grunes, and P. L. Fejes, Phys. Rev. B 26, 614 (1982).

28 J. Fink, Th. Müller-Heinzerling, B. Scheerer, W. Speier, F. U. Hillebrecht, J. C. Fuggle, J. Zaanen, and G. A. Sawatzky, Phys. Rev. B 32, 4899 (1985).

29 J. Zaanen, G. Sawatzky, J. Fink, W. Speier, and J. C. Fuggle, Phys. Rev. B 32, 4905 (1985).

${ }^{30}$ J. Barth, F. Gerken, and C. Kunz, Phys. Rev. B 28, 3608 (1983).

${ }^{31}$ J. Pflüger, Kernforschungszentrum Karlsruhe Internal Report No. KfK-3585, 1983 (unpublished), p. 70.

${ }^{32}$ D. K. G. de Boer, C. Haas, and G. A. Sawatzky, J. Phys. F 14, 2769 (1984).

${ }^{33} \mathrm{P}$. Hedegård and F. U. Hillebrecht, Nordisk Institut for Teoretisk Atomfysik Report No. NORDITA-85/38, 1985 (unpublished). 